
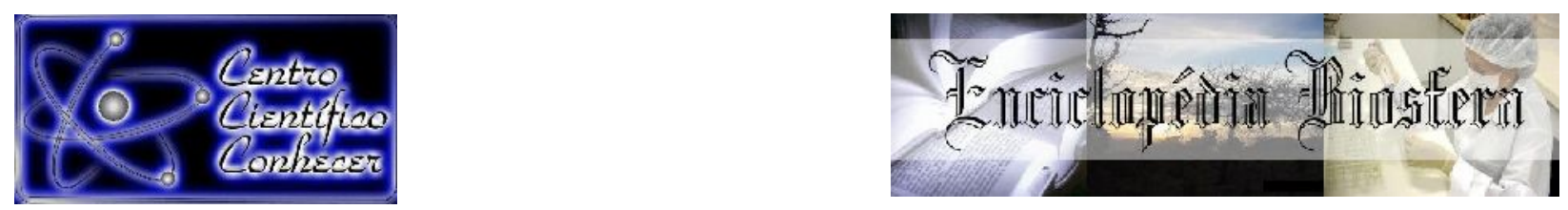

\title{
INVASÃO DE BAMBU DOURADO (PHYLLOSTACHYS AUREA) ALTERA A ESTRUTURA E A DIVERSIDADE DA REGENERAÇÃO NATURAL EM UM FRAGMENTO FLORESTAL URBANO LOCALIZADO EM CURITIBA, PR
}

\author{
Román Carlos Rios ${ }^{1}$, Guilherme Henrique Farias ${ }^{2}$ \\ ${ }^{1}$ Doutor em Engenharia Florestal pela UFPR, área de Conservação da Natureza. \\ Professor Adjunto na UFPR no Curso de Engenharia Florestal. \\ Email: rioselvas@gmail.com \\ ${ }^{2}$ Mestrando em Ciências Florestais pela UFRPE na linha de Ecologia e Conservação \\ da Natureza. \\ Recebido em: 15/08/2021 - Aprovado em: 15/09/2021 - Publicado em: 30/09/2021 \\ DOI: 10.18677/EnciBio_2021C26 \\ trabalho licenciado sob licença Creative Commons Attribution-NonCommercial-NoDerivatives 4.0 International License.
}

\begin{abstract}
RESUMO
As espécies de bambu com alto poder de invasão tem sido especialmente bemsucedidas em vários países do mundo competindo com a vegetação nativa. Assim Phyllostachys aurea é uma das espécies mais agressivas e de difícil controle com escassos estudos sobre seus impactos negativos na estrutura da vegetação nativa. Objetivou-se com a realização deste trabalho avaliar o efeito do estabelecimento e o avanço do bambu dourado na borda de um fragmento de Floresta Ombrófila Mista em Curitiba. Foram instaladas 34 parcelas em cinco linhas paralelas à borda e consecutivas para o interior do fragmento. Foram medidos os exemplares arbóreos regenerantes de $10 \mathrm{~cm}$ de altura até $5 \mathrm{~cm}$ de diâmetro, o diâmetro do bambu à altura do colo, a luminosidade média incidente em cada parcela e a altura dos regenerantes. A densidade do bambu decresce da borda para o interior do fragmento e altera a composição, a riqueza, a altura e a diversidade verdadeira da regeneração natural, parâmetros que aumentam para o interior. $O$ bambu dourado emite rizomas para 0 interior da floresta sem sucesso no estabelecimento. Conclui-se que a 0 estabelecimento de Phyllostachys aurea em um fragmento urbano localizado em Curitiba altera a estrutura, a composição e a diversidade da regeneração natural e o normal desenvolvimento da sucessão secundária da Floresta Ombrófila Mista.
\end{abstract}

PALAVRAS-CHAVE: Floresta Ombrófila Mista; Diversidade verdadeira; Sucessão.

\section{GOLDEN BAMBOO (PHYLLOSTACHYS AUREA) INVASION CHANGES THE STRUCTURE AND DIVERSITY OF NATURAL REGENERATION IN AN URBAN FOREST FRAGMENT LOCATED IN CURITIBA, PR}

\begin{abstract}
Bamboo species with high invasive power have been especially successful in several countries around the world, competing with native vegetation. Thus, Phyllostachys aurea is one of the most aggressive and difficult to control species with few studies on its negative impacts on the structure of native vegetation. The objective of this work was to evaluate the effect of establishment and advancement of golden bamboo on the edge of a fragment of Mixed Ombrophilous Forest in Curitiba. 34 plots were installed in five lines parallel to the edge and consecutive to the interior of the fragment. The regenerating tree specimens from $10 \mathrm{~cm}$ in height to $5 \mathrm{~cm}$ in diameter, the bamboo
\end{abstract}


diameter at the height of the collar, the average luminosity incident on each plot and the height of the regenerants were measured. Bamboo density decreases from the edge to the interior of the fragment and alters the composition, richness, height and true diversity of natural regeneration, parameters that increase towards the interior. Golden bamboo emits rhizomes into the forest without success in the establishment. It is concluded that the establishment of Phyllostachys aurea in an urban fragment located in Curitiba alters the structure, composition and diversity of natural regeneration and the normal development of secondary succession in the Mixed Ombrophilous Forest.

KEYWORDS: Mixed Ombrophylous Forest; True diversity; Succession.

\section{INTRODUÇÃO}

As plantas exóticas invasoras estão sendo consideradas seriamente ao nível mundial pelas ameaças concretas à biodiversidade local e à continuidade dos serviços ecossistêmicos brindados pela vegetação nativa. A invasão biológica é o resultado das intervenções humanas e segundo Canavan et al., (2016) podem ser compreendidas no contexto das atividades dessa espécie.

A subfamília Bambusoidea (Poaceae) com 121 gêneros e 1662 espécies, distribui-se por numerosas regiões biogeográficas do mundo formando parte da cultura dos povos locais (CANAVAN et al., 2016). Dados os numerosos usos comerciais do bambu, a maioria dos estudos estão focados nesse sentido e muito escassos estudos fazem referência ao potencial invasor das 12 espécies com essas características.

O género Phyllostachys com 59 espécies tem 24 espécies que foram distribuídas fora de sua região de origem. Dessas, seis são listadas como invasoras e com alto poder de competir com as espécies nativas de forma muito agressiva (LIEURANCE et al., 2018; XU et al., 2020). O bambu dourado (Phyllostachys aurea Carrière ex Rivière \& $\mathrm{C}$. Rivière) foi introduzido no Brasil como planta ornamental e como provedora de produtos e materiais de construção (SETTE Jr et al., 2017; DOLCI DE CARVALHO et al., 2020).

$\mathrm{Na}$ atualidade a espécie está distribuída em numerosos estados da área de influência da Mata Atlântica, no entanto, o seu impacto negativo sobre os fragmentos florestais remanescentes são muito escassos. A nível governamental, tanto nacional como estadual têm-se tomado medidas institucionais para realizar ações tendentes a minimizar o problema das espécies exóticas invasoras. Assim o estado do Paraná oficializou a lista de espécies exóticas invasoras por meio da portaria $n^{\circ} 125 / 2009$ em que Phyllostachys aurea foi classificada na categoria I que é a mais restritiva pois proíbe o plantio e o uso da espécie (INSTITUTO AMBIENTAL DO PARANÁ, 2009).

Os efeitos da invasão do bambu dourado sobre os fragmentos florestais urbanos das grandes cidades do sul do Brasil não foram avaliados até o presente momento. Essa espécie tem como principais características o alto poder de expansão de rizomas (caules leptomorfos), alto nível de crescimento e demanda por grande quantidade de luminosidade. Isso a faz muito eficiente na invasão de bordas de florestas urbanas onde podem permanecer por décadas com o potencial de avançar em clareiras ou áreas com distúrbios antrópicos. Assim, neste trabalho será estudado os impactos da invasão do bambu dourado num fragmento florestal urbano de Curitiba localizado no Campus III da UFPR.

Formulou-se os seguintes objetivos: 1) avaliar as alterações que provocam a invasão do bambu dourado na estrutura, composição e a diversidade da regeneração natural. 2) determinar a capacidade do bambu dourado de penetrar com sucesso no 
sub-bosque da Floresta Ombrófila Mista da área estudada. 3) pesquisar o grau de alteração à sucessão secundária pela presença do bambu dourado.

\section{Área de estudo}

\section{MATERIAL E MÉTODOS}

O estudo foi desenvolvido em um fragmento florestal urbano inserido no Campus III da Universidade Federal do Paraná (UFPR) e que é conhecido como capão da engenharia florestal. Com uma área total de 15 ha, o capão está localizado nas coordenadas. 2526'50" e 2527'33"S e 4914'16" e 4914'33"W e 900 m de altitude numa matriz urbana muito adensada (Figura $1 \mathrm{~A}$ ). Segundo classificação de Koppën, o clima é classificado como Cfb: subtropical úmido mesotérmico de verões frescos, inverno com geadas frequentes. A precipitação média varia entre 1300 a $1500 \mathrm{~mm}$ (RONDON NETO et al., 2002).

A vegetação e Floresta Ombrófila Mista montana (IBGE, 2012) tem predomínio das seguintes espécies: Araucaria angustifolia (Bertoloni) Otto Kuntze, Ocotea porosa (Nees \& C. Mart.) Barroso, O. puberula (Rich.) Nees, O. pulchella (Lauraceae), Capsicodendron dinisii (Schwacke) Occhioni (Canellaceae), Moquiniastrum polymorphum (Less.) G. Sancho (Asteraceae), Podocarpus lambertii Klotzsch ex Eichler (Podocarpaceae), Ilex paraguariensis A.St.-Hil (Aquifoliaceae),Cedrela fissilis Vell (Meliaceae), Campomanesia xanthocarpa O. Berg (Myrtaceae), Matayba elaeagnoides Radlk. (Sapindaceae), Sloanea lasiocoma K. Schum. (Elaeocarpaceae), Luehea divaricata Mart. (Malvaceae), Mimosa scabrella Benth. (Fabaceae), Dalbergia brasiliensis Vogel (Fabaceae), Jacaranda puberula Cham. e Handroanthus albus (Cham.) Mattos (Bignoniaceae).

FIGURA 1 - Localização do capão da Engenharia Florestal no Campus III da UFPR, Curitiba, Paraná. A: Matriz urbana onde se incerta a área de estudo. B: Vista superior. C: Disposição das linhas de parcelas e os círculos amarelos representam a proporção de bambu dourado.
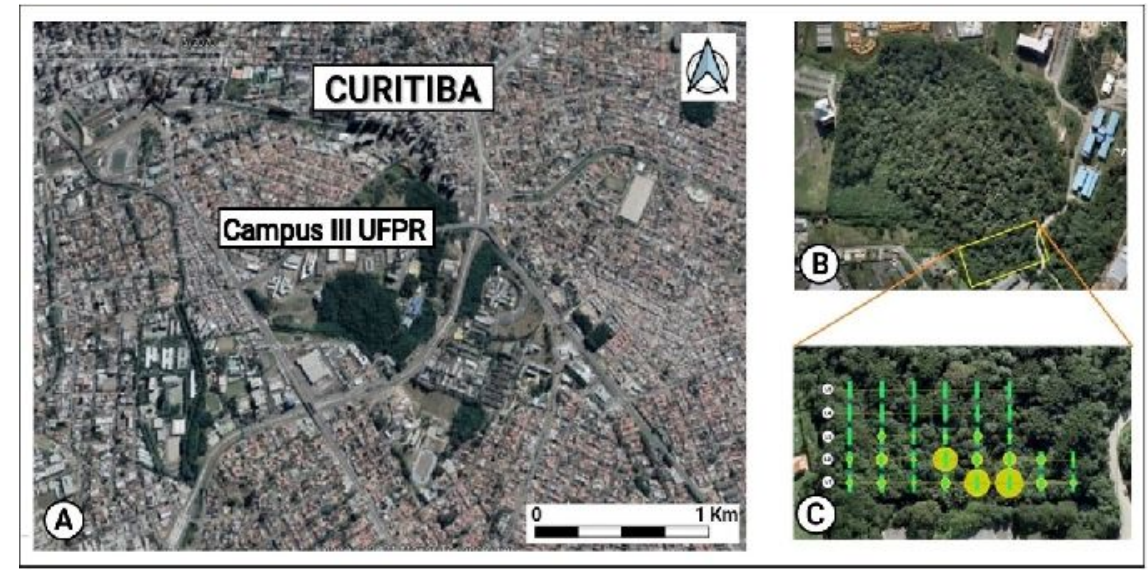

Fonte: Os autores (2021), Google Earth (2021)

\section{Coleta dos dados}

Foram instaladas 34 parcelas permanentes de $10 \mathrm{~m}^{2}$ em cinco linhas paralelas à borda e consecutivas para o interior do fragmento (Figura $1 \mathrm{~B}$ e C). Foram mensurados os exemplares de bambu dourado com calibre à altura do colo, classificando os em vivos e mortos. A regeneração natural de árvores foi registrada mediante a mediação de alturas com régua graduada e vara telescópica. A 
luminosidade média incidente em cada parcela foi registrada com luxímetro Lutron escala de 0 a 50000 lux. Foram realizadas duas leituras por parcelas entre as 11 e 13 horas da tarde em dias de céu azul. A identificação das espécies foi realizada com consulta a bibliografia especializada e consulta a especialistas.

\section{Análise dos dados}

Para testar a variação da superfície transversal do bambu dourado entre as linhas, a verificação de diferenças na luminosidade incidente nas parcelas e a abundância entre as linhas foi usado o teste Kruskal-Wallis uma vez que os dados não respondem aos pressupostos para testes paramétricos. O teste qui quadrado foi aplicado para detectar diferença entre colmos de bambu dourado vivos e mortos entre as linhas.

Para avaliar as diferenças em riqueza e altura da regeneração natural foi usado o teste paramétrico ANOVA com o teste a posteriori Tukey, com a verificação de pressupostos de homogeneidade e normalidade com os testes de Levene e Shapiro Wilks respectivamente.

A diversidade verdadeira foi avaliada segundo o método proposto por Chão e Jost (2012) com o uso dos pacotes iNEXT (HSIEH et al., 2016) e ggplot2 (WICKHAM, 2016) para o programa $R$ ( $R$ CORE TEAM, 2021) que possibilitam a visualização gráfica dos valores $\mathrm{q}=0, \mathrm{q}=1$ e $\mathrm{q}=2$, que permitem a comparação da diversidade segundo a abundância das espécies. Assim q=0 não considera a abundância, avalia somente a riqueza de espécies.

A diversidade verdadeira das espécies menos frequentes é comparada por $q=1$ e as espécies mais abundantes são comparadas com $q=2$ (CHAO et al., 2015). A sobreposição ou não dos intervalos de confiança permitem estabelecer as diferenças entre linhas de parcelas (COLWELL et al., 2012).

A similaridade em abundância e composição entre as linhas foi avaliada com o índice de similaridade de Bray-Curtis e a análise NMDS com a aplicação de teste PERMANOVA para verificar as diferenças estatísticas entre as linhas de parcelas. Para o cálculo do Percentual de Importância e para a construção da tabela fitossociológica calcularam-se a densidade absoluta e relativa (DA e DR), e frequência absoluta e relativa (FA e FR) (MUELLER-DOMBOIS; ELLEMBERG, 1974).

\section{RESULTADOS}

A densidade de colmos de bambu dourado foi de $13.970 \mathrm{ind} / \mathrm{ha}$ e a superfície transversal por parcela foi diferente entre as linhas sendo maior nas mais afastadas da borda. Segundo os testes Kruskal-Wallis $\left(X^{2}=43,2 ; d f=4 ; p=9,37 e^{-09}\right)$ e o teste Bonferroni as linhas L1, L2 e L3 foram similares e diferiram das linhas L4 e L5. Considerando os colmos de bambu vivos e mortos mostraram diferenças significativas entre as linhas L1, L2 e L3 em relação às linhas L4 e L5 mediante o teste qui quadrado $\left(X^{2}=16,4 ; d f=4, p=0,0024\right)$ com predomínio de colmos vivos perto da borda (Figura 1 C, Figura 2). A luminosidade média incidente nas parcelas não diferiu significativamente segundo o teste Kruskal-Wallis $\left(X^{2}=6,30 ; d f=4 ; p=0,177\right)$ em função das linhas distantes da borda.

Foram registradas 61 espécies arbóreas regenerantes e considerando a riqueza de espécies nas linhas a partir da borda para o interior teve-se: L1 (40), L2 (38), L3 (42), L4 (37) e L5 (45). Sendo a riqueza estatisticamente diferentes as linhas L2 e L5 (Fig. 3) segundo o teste paramétrico ANOVA ( $F=6,98 ; p=0,0004)$ e com o teste de Tukey. Considerando a similaridade em composição florística vê-se na Figura 4 que as linhas L1 e L2 são similares entre si e diferem das linhas L4 e L5 e são 
estatisticamente diferentes segundo a análise PERMANOVA ( $F=2,33 ; p=0,001)$. Em relação a abundância ou número de indivíduos por parcela não apresentou diferenças significativas segundo o teste Kruskal-Wallis $\left(X^{2}=3,59 ; d f=4 ; p=0,465\right)$.

FIGURA 2: Perfil da área de estudo. Espécies: 1. Symplocos tetrandra; 2. Nectandra lanceolata; 3. Ocotea nutans; 4. Ocotea puberula; 5. Ilex paraguariensis; 6. Luehea divaricata; 7. Citronela paniculata; 8. Casearia obliqua; 9. Casearia sylvestris; 10. Eugenia uniflora; 11. Ocotea bicolor; 12. Araucaria angustifolia; Bu. Phyllostachys aurea.

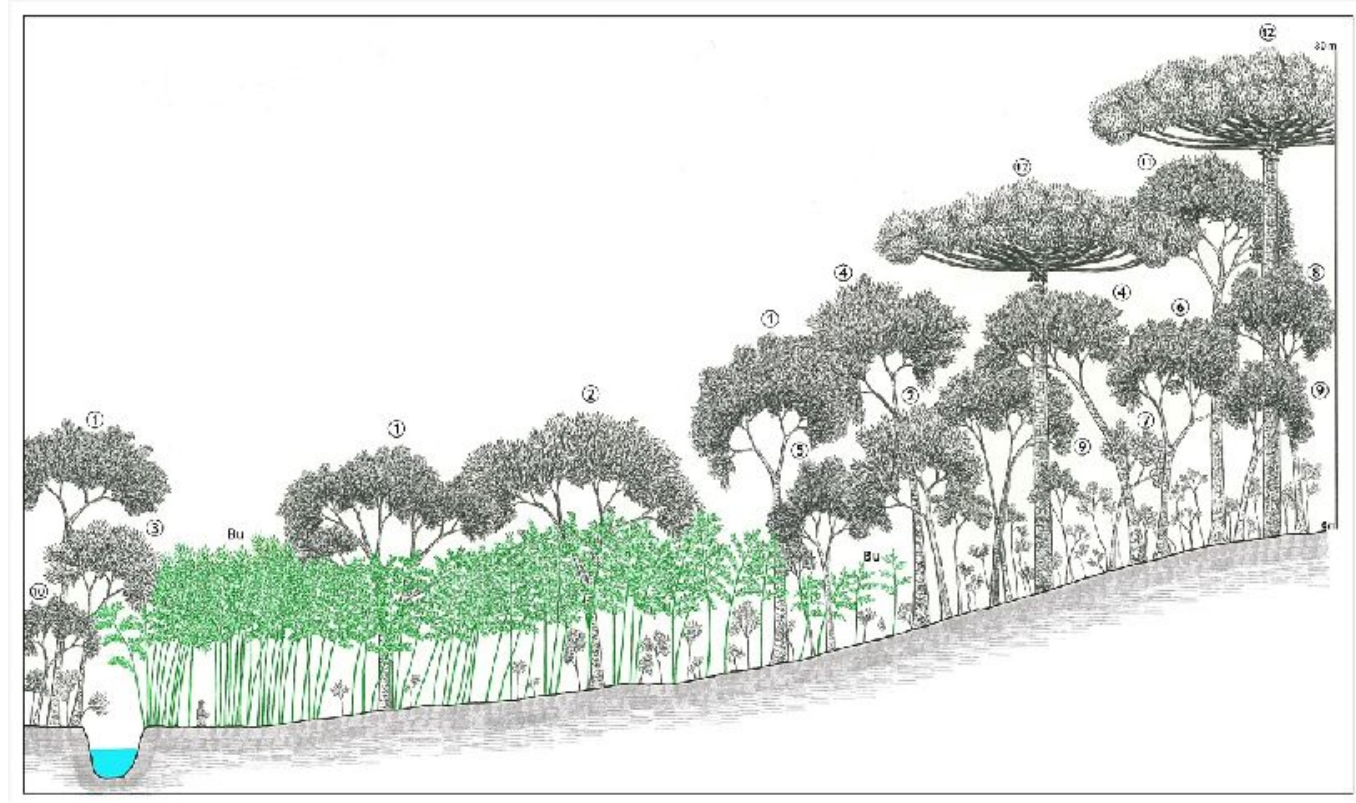

Fonte: Ríos (2021).

Considerando a diversidade verdadeira de ordem ( $q=1$ ) (Figura 3) observaramse diferenças significativas entre as linhas $L 1$ e $L 2$ em relação às linhas mais internas na floresta (L3, L4 e L5). Quando foi observada a diversidade verdadeira de ordem $(\mathrm{q}=2)$ teve-se o mesmo resultado com as espécies mais abundantes melhor distribuídas nas linhas L3, L4 e L5. As linhas L1 e L2 apresentaram distribuição menos equitativa entre as espécies (Figura 3). Comparando as alturas dos regenerantes observaram-se diferenças significativas segundo o teste ANOVA $(F=15,49 ; d f=4 ; p=$ $6,88 \mathrm{e}^{-07}$ ) e o teste de Tukey mostrou que as linhas internas L4 e L5 apresentaram maiores alturas (Figura 4). 
FIGURA 3 - Diversidade verdadeira nas cinco linhas de parcela: $q=$ 0 (riqueza de espécies), $q=1$ e $q=2$ (diversidade segundo a distribuição de abundância), no capão da Engenharia Florestal da UFPR, Curitiba, Paraná.

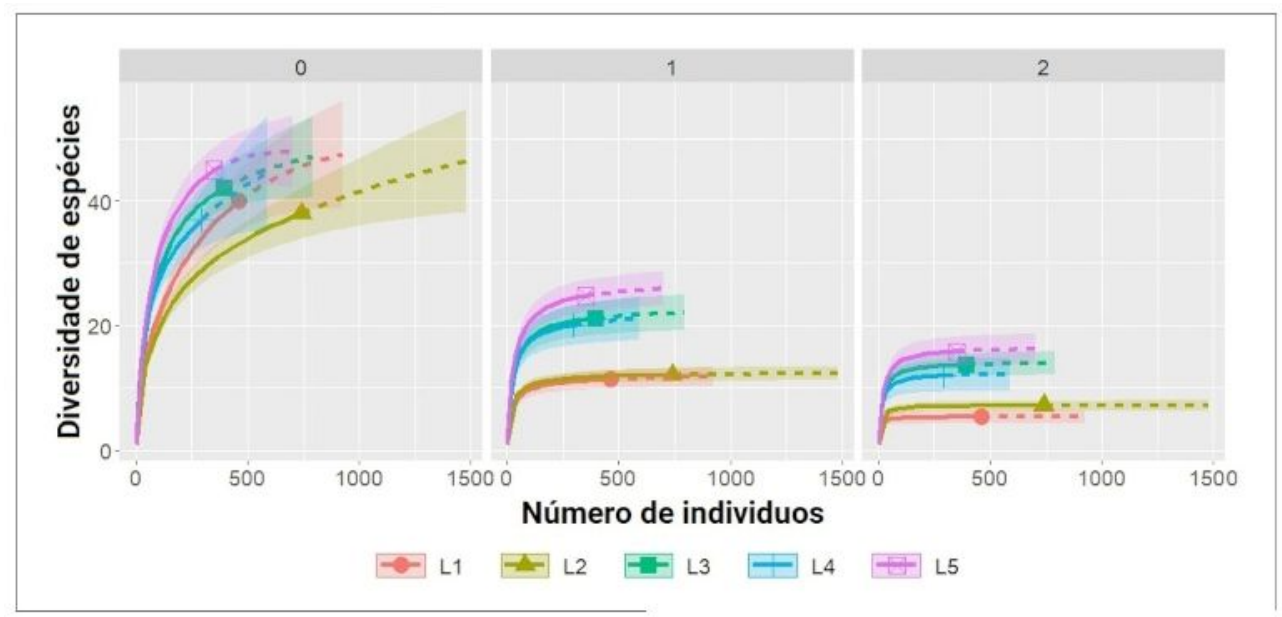

Fonte: Os autores (2021).

FIGURA 4 - A: Distribuição das parcelas em função da similaridade florística entre as linhas. Valor de estresse $=0,18$. B: Altura da regeneração natural segundo as linhas de parcelas no capão da Engenharia Florestal da UFPR, Curitiba, Paraná.

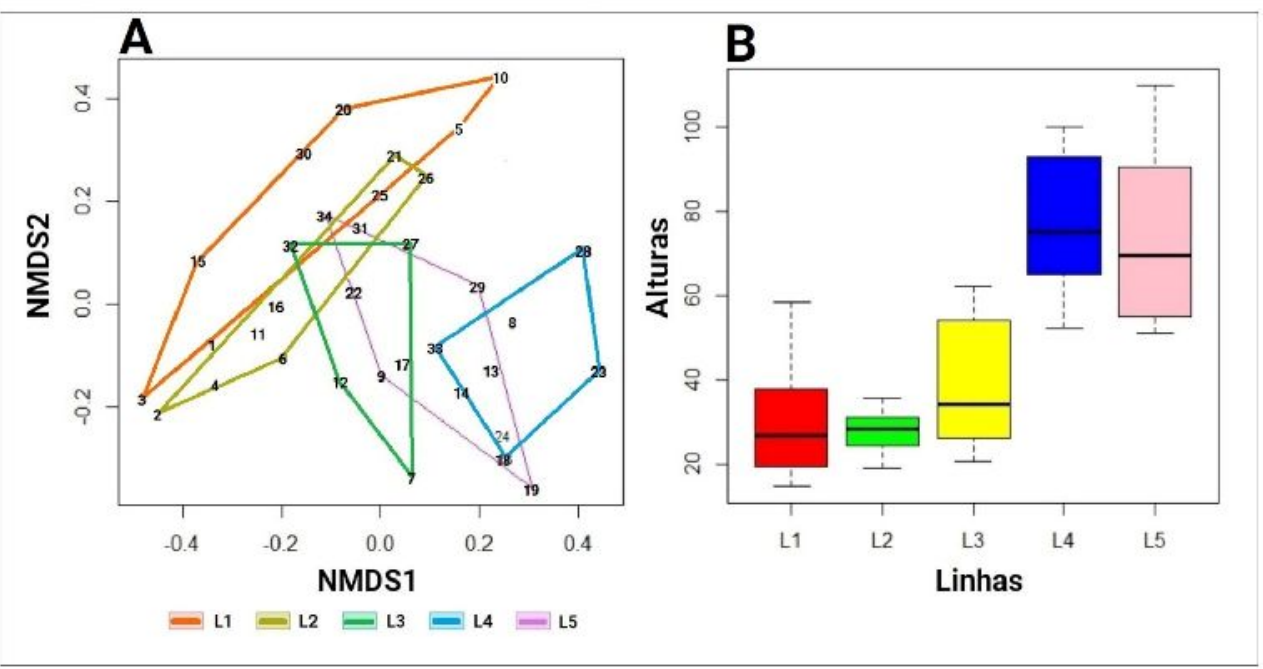

Fonte: Os autores (2021).

Comparando as 15 principais espécies em dois grupos em função da localização, um de borda constituído pelas linhas L1 e L2 e o grupo do interior conformado pelas linhas L4 e L5. O grupo da borda apresentou alta densidade de colmos de bambu dourado (grupo bambu). O grupo do interior apresentou baixa densidade de bambu dourado (grupo sem bambu).

Assim, considerando o percentual de importância do grupo bambu, apresentou a espécie Symplocos tetrandra (19,69\%) com maior importância ecológica seguida de Allophylus edulis (8,76\%), Mollinedia clavigera (7,93\%), Myrcia hastchbachii (6,60\%), 
Ocotea puberula (6.18\%), Allophylus semidentata (5,86\%), Eugenia uniflora $(4,75 \%)$, Eriobotrya japônica (4,66\%), Myrsine coriaceae $(3,02 \%)$, dentre outras (Tabela 1). No grupo sem bambu observou-se como principal espécie a Myrceugenia acutiflora $(12,02 \%)$, seguida de Allophylus semidentatus $(7,22 \%)$, Mollinedia clavigera $(5,06 \%)$, Myrceugenia miersiana (4,83\%), Myrcia hatschbachii (4,79\%), Casearia decandra $(4,14 \%)$, Cupania vernalis (3,68\%), Sloanea monosperma (3,60\%), Allophylus edulis $(3,31 \%)$, Ocotea puberula $(3,15 \%)$, dentre outras (Tabela 1). Quando considerado o número de indivíduos, teve-se que as 15 principais do grupo bambu totalizou $90,59 \%$ e o grupo sem bambu somou $75,85 \%$. Pode-se afirmar que essas espécies são as mais comuns em ambos os grupos.

TABELA 1 - Estimativas dos parâmetros fitossociológicos das 15 espécies com maior percentual de importância da regeneração natural (PI) no capão da Engenharia Florestal da UFPR, Curitiba, Paraná.

\section{Espécie}

Symplocos tetrandra Mart.

Allophylus edulis (A.St.-Hil. et al.) Hieron. ex Niederl.

Mollinedia clavigera Tul.

Myrcia hatschbachi D.Legrand

Ocotea puberula (Rich.) Nees

Allophylus semidentatus (Miq.) Radlk.

Eugenia uniflora L.

Eriobotrya japonica (Thunb.) Lindl.

Myrsine coriacea (Sw.) R.Br. ex Roem. \& Schult.

Casearia decandra Jacq.

Ocotea bicolor Vattimo-Gil

Cordiera concolor (Cham.) Kuntze

Ocotea nutans (Nees) Mez

Cedrela fissilis Vell.

Prunus brasiliensis (Cham. \& Schltdl.) D.Dietr.

\section{Espécie}

Myrceugenia acutiflora (Kiaersk.) D.Legrand \& Kausel

Allophylus semidentatus (Miq.) Radlk.

Mollinedia clavigera Tul.

Myrceugenia miersiana (Gardner) D.Legrand \& Kausel

Myrcia hatschbachii D.Legrand

Casearia decandra Jacq.

Cupania vernalis Cambess.

Sloanea monosperma Vell.

Allophylus edulis (A.St.-Hil. et al.) Hieron. ex Niederl.

Ocotea puberula (Rich.) Nees

Eugenia uniflora L.

\begin{tabular}{cccc}
\multicolumn{4}{c}{ Parcelas com bambu dourado } \\
DA & FR & DR & PI \\
25375 & 5.603 & 33.78 & 19.69 \\
8312.5 & 6.466 & 11.06 & 8.7652 \\
7062.5 & 6.466 & 9.401 & 7.9333 \\
5062.5 & 6.466 & 6.739 & 6.6021 \\
4437.5 & 6.466 & 5.907 & 6.1862 \\
5250 & 4.741 & 6.988 & 5.8649 \\
2937.5 & 5.603 & 3.91 & 4.7568 \\
3125 & 5.172 & 4.16 & 4.6661 \\
1625 & 3.879 & 2.163 & 3.0212 \\
1062.5 & 3.448 & 1.414 & 2.4313 \\
687.5 & 3.448 & 0.915 & 2.1817 \\
937.5 & 2.586 & 1.248 & 1.9171 \\
687.5 & 2.586 & 0.915 & 1.7507 \\
562.5 & 2.155 & 0.749 & 1.452 \\
562.5 & 2.155 & 0.749 & 1.452
\end{tabular}

Parcelas sem bambu dourado

$\begin{array}{cccc}\text { DA } & \text { FR } & \text { DR } & \text { PI } \\ 10333 & 4.846 & 19.2 & 12.02 \\ 5166.7 & 4.846 & 9.598 & 7.2217 \\ 3083.3 & 4.405 & 5.728 & 5.0664 \\ 2833.3 & 4.405 & 5.263 & 4.8342 \\ 3500 & 3.084 & 6.502 & 4.7926 \\ 2333.3 & 3.965 & 4.334 & 4.1496 \\ 1833.3 & 3.965 & 3.406 & 3.6852 \\ 1750 & 3.965 & 3.251 & 3.6078 \\ 1666.7 & 3.524 & 3.096 & 3.3101 \\ 1500 & 3.524 & 2.786 & 3.1553 \\ 1666.7 & 3.084 & 3.096 & 3.0898\end{array}$


Prunus brasiliensis (Cham. \& Schltdl.) D.Dietr.

$\begin{array}{llll}1583.3 & 3.084 & 2.941 & 3.0124\end{array}$

Ocotea bicolor Vattimo-Gil

$\begin{array}{llll}1333.3 & 3.524 & 2.477 & 3.0005\end{array}$

Symplocos tetrandra Mart.

1333.3

$\begin{array}{lll}2.643 & 2.477 \quad 2.56\end{array}$

Machaerium stipitatum Vogel

916.67

$\begin{array}{lll}2.643 & 1.703 & 2.173\end{array}$

Onde: DR - Densidade relativa (\%), FR = frequência relativa (\%), PI - Porcentual de Importância (\%). Fonte: Os autores (2021)

\section{DISCUSSÃO}

O bambu dourado se estabeleceu na área de estudo faz mais de 20 anos logo após finalizada a tarefa de retificação do rio que faz parte do limite sul do capão (Figura 2). O espaço aberto pelas atividades mencionadas foi rapidamente ocupado pelo bambu dourado com as maiores densidades na borda propiciada pela exposição solar. O gênero Phyllostachys, dada a sua elevada taxa de crescimento, que pode ser de mais de um metro por dia (BAl et al., 2016), precisa de grande quantidade de luminosidade. Isso explicaria porque as linhas sem bambu e já no interior da floresta com dossel alto e denso apresenta um número inexpressivo de colmos de bambu vivos e alta frequência de colmos mortos.

A alta concentração de bambu dourado na borda induz a uma composição de espécie diferenciada em relação às parcelas das linhas interiores no capão como observado por Tian et al., (2020), e que também pode estar influenciada pela chuva de sementes no local. Assim, na borda, onde existem algumas árvores remanescentes adultas de Symplocos tetrandra (Symplocaceae), observou-se o predomínio dessa espécie na regeneração natural. Nas linhas do interior a espécie predominante é Myrceugenia acutiflora (Myrtaceae) que é uma espécie clímax de sub-bosque. A similaridade é baixa segundo o índice Bray-Curtis que também leva em consideração as densidades das espécies. Isto poderia indicar que o bambu dourado altera as condições ambientais como as propriedades físico-químicas (WANG et al., 2018), disponibilidade de elementos como C e N (BAl et al., 2016; TIAN et al., 2020) e a composição e diversidade de micro-organismos (INDERJET et al., 2010).

A maior presença de bambu na borda provoca a diminuição da diversidade verdadeira refletida na distribuição da abundância, tanto nas espécies raras como nas mais comuns (BAl et al., 2016b; KUDO et al., 2017). Essa situação é similar ao que acontece com a invasão de Phyllostachys edulis na China e com alguns bambus nativos como Guadua tagoara (LIMA et al., 2012).

Ao comparar as alturas dos regenerantes segundo as linhas tem-se marcada diferença com exemplares mais altos nas linhas internas (L4 e L5). Com similar abundância, pode-se inferir que na borda existe germinação e estabelecimento de plântulas, porém, não há condições para o crescimento em altura devido a alta mortalidade (LARPKERN et al., 2011). Lima et al., (2012) obtiveram resultados similares em parcelas com e sem bambu nativo em estudo realizado no estado de São Paulo.

São escassos os estudos no Brasil sobre o bambu dourado e sua relação com a regeneração da floresta e existem diversos estudos das propriedades físico-químicas dos colmos como material industrial e sobre controle mecânico das populações (DOLCI DE CARVALHO et al., 2020). No estado do Paraná o bambu dourado está na lista de espécies exóticas invasoras com a categoria I que é de total proibição de plantio e uso (INSTITUTO AMBIENTAL DO PARANÁ, 2009). Porém, a espécie já está estabelecida em numerosas áreas e muitas em estreito contato com florestas urbanas. 
Como mostrado neste trabalho o bambu dourado tem a capacidade de se instalar num local e afetar a regeneração natural arbórea e portanto impedir o normal desenvolvimento da sucessão natural como discutido por Larpkern et al., (2011). Assim, na área de estudo o bambu dourado colonizou e se estabeleceu em área que tinha que ser ocupada pela sucessão secundária da Floresta Ombrófila Mista. Não foi demonstrado que o bambu penetra na floresta, e se estabelece com sucesso, porém, foi demonstrado que os rizomas avançam para o interior e que os colmos acabam morrendo por falta de luz. Essa é uma diferença marcante com Phyllostachys edulis que pode avançar no interior da floresta subtropical do sul da China e pode competir com a regeneração natural (ZHANG;XUE, 2018).

Segundo Spake et al., (2021), para poder prevenir e controlar os impactos negativos da invasão biológica necessita-se conhecer os fatores de sucesso dessa invasão. A hipótese de gradiente de estresse (SGH) brinda um marco conceitual que permite explicar como o contexto ambiental determina que as espécies nativas inibidoras ou facilitadoras da invasão (SPAKE et al., 2021). Estudos com o género Phyllostachys tem demonstrado que a fotoinibição é potencializada pela sombra do dossel florestal em regiões de clima subtropical (LOU et al., 2018; SPAKE et al., 2021). Assim Phyllostachys aurea na área de estudo responde perfeitamente à hipótese de gradiente de estresse dado que não consegue se estabelecer na sombra do dossel formado pelas copas das árvores da floresta.

Em função do longo ciclo de vida dos Phyllostachys no geral, tem-se uma situação preocupante que é da presença do bambu dourado que permanecerá por mais de 100 anos emitindo rizomas para o interior da floresta. Este longo tempo de espera vai permitir ao bambu dourado invadir clareiras grandes que se formarão naturalmente.

A invasão vai ser de longo prazo, potenciada pela permanente alteração do sob-bosque da floresta pela coleta sistemática de pinhão e pela perda de fauna silvestre provocada pelo ingresso de animais domésticos caçadores (cães) (BAl et al., 2016). Qualquer alteração drástica do dossel da floresta vai ser uma oportunidade para a invasão do bambu que já está instalado em cerca de $10 \%$ do perímetro do capão.

A remoção total ou o controle físico para evitar o avanço dos rizomas é de fundamental importância para evitar a descaracterização de um dos poucos fragmentos de Floresta Ombrófila Mista urbana de Curitiba. $O$ aumento do sombreamento dado pelo dossel florestal limita a expansão do bambu dourado (SPAKE et al., 2021). São necessários estudos e experimentos que permitam o delineamento de ações de manejo para a recuperação do dossel das florestas secundárias urbanas onde o bambu dourado apresenta seu maior potencial de invasão.

\section{CONCLUSÕES}

A presença do bambu dourado na borda do capão da Engenharia Florestal da UFPR alterou a estrutura e a composição e a diversidade da regeneração natural. Foi detectada predominância de superfície transversal do bambu dourado na borda do capão o que afeta o conjunto de espécies presentes nesta área e induzem a uma menor altura da regeneração natural o que indicaria um atraso no estabelecimento e no desenvolvimento normal da sucessão secundária.

O bambu dourado emite rizomas para o interior da floresta, porém sem sucesso dado a alta mortalidade de colmos o que descartaria a capacidade de invasão dentro do sub-bosque de uma floresta fechada (madura). O bambu dourado representa uma 
ameaça real e potencial muito grande visto que pode permanecer atrasando a sucessão natural e aguardando uma oportunidade de ingressar na floresta por mais de 100 anos.

\section{REFERÊNCIAS}

BAI, S.B.; WANG, Y.X.; CONANT, R.T.; ZHOU, G.; XU Y.; WANG, N.; FANG, F.; CHEN, J. Can native clonal moso bamboo encroach on adjacent natural forest without human intervention? Scientific Reports 6:31504. 2016. DOI: 10.1038/srep31504.

BAI, S.; CONANT, R. T.; ZHOU, G.; WANG, Y.; WANG, N.; LI Y.; ZHANG, K. Effects of moso bamboo encroachment into native, broadleaved forests on soil carbon and nitrogen pools. Scientific Reports 6:31480. 2016. DOI: 10.1038/srep31480

CANAVAN, S.; RICHARDSON, D. M.; VISSER, V.; LE ROUX, J. J.; VORONTSOVA, M. S.; WILSON, J. R. U. 2017. The global distribution of bamboos: assessing correlates of introduction and invasion. AoB PLANTS 9: plw078; doi:10.1093/aobpla/plw078

CHAO, A.; JOST, L. Coverage-based rarefaction and extrapolation: standardizing samples by completeness rather than size. Ecology, 93, n.12, p.2533-2547, 2012. DOI: https://doi.org/10.1890/11-1952.1.

CHAO, A.; CHIU, C. H.; HSIEH, T. C.; DAVIS, T.; NIPPERESS, D. A.; FAITH, D. P. Rarefaction and extrapolation of phylogenetic diversity. Methods in Ecology and Evolution, v.6, p.380-388, 2015. DOI: https://doi.org/10.1111/2041-210X.12247.

COLWELL, R.K.; CHAO, A.; GOTELLI, N.J.; LIN, S.-Y.; MAO, C.X.; CHAZDON, R.L.; LONGINO, J.T. Models and estimators linking individual-based and sample-based rarefaction, extrapolation and comparison of assemblages. Journal of Plant Ecology, v.5, p.3-21, 2012. DOI: https://doi.org/10.1093/jpe/rtr044.

DOLCI DE CARVALHO, M. C.; SAQUETTA, C. R.; DALlA CORTE, A. P. Efeitos do controle mecânico sobre Phyllostachys aurea Carr. ex A. \& C. Rivière no Parque Estadual de Vila Velha - PR. Ciência Florestal, Santa Maria, v. 30, n. 3, p. 907-915, jul./set. 2020. DOI: https://doi.org/10.5902/1980509837089

HSIEH, T. C.; MA, K. H.; CHAO, A. iNEXT: an R package for rarefaction and extrapolation of species diversity (Hill numbers). Methods in Ecology and Evolution, v. 7, p.1451-1456, 2016. DOI: https://doi.org/10.1111/2041-210X.12613.

INSTITUTO AMBIENTAL DO PARANÁ. Portaria no 125 de 07 de agosto de 2009. Reconhece a Lista Oficial de Espécies Exóticas Invasoras para o Estado do Paraná, estabelece normas de controle e dá outras providências. Disponível em: http://www.iap.pr.gov.br/arquivos/File/Legislacao_ambiental/Legislacao_estadual/POR TARIAS/PORTARIA_IAP_125_2009_ESPECIES_EXOTICAS.pdf 
IBGE (2012). Manual técnico da vegetação brasileira. Instituto Brasileiro de Geografia e Estatística, Coordenação de Recursos Naturais e Estudos Ambientais, Rio de Janeiro, Brazil, 272. Available at $<$ https://biblioteca.ibge.gov.br/index.php/bibliotecacatalogo?view=detalhes\&id=263011 > . Access on: 10 April 2021.

KUDO, G.; KAWAI, Y.; AMAGAI, Y. Degradation and recovery of an alpine plant community: experimental removal of an encroaching dwarf bamboo. Alp Botany 127, 75-83 (2017). https://doi.org/10.1007/s00035-016-0178-2

LARPKERN, P.; MOE, S.R.; TOTLAND, $\varnothing$. Bamboo dominance reduces tree regeneration in a disturbed tropical forest. O ecologia 165, 161-168. 2011. https://doi.org/10.1007/s00442-010-1707-0

LIMA, R.A.F.; ROTHER, D. C.; MULER, A. E.; LEPSCH, I. F.; RODRIGUES, R. R. Bamboo overabundance alters forest structure and dynamics in the Atlantic Forest $\begin{array}{lllll}\text { hotspot. Biological } & \text { Conservation } & 147 & \text { (2012) } & \text { 32-39. }\end{array}$ doi:10.1016/j.biocon.2012.01.015

LIEURANCE, D.; COOPER, A.; YOUNG, A. L.; GORDON, D. R.; FLORY, S. L. Running bamboo species pose a greater invasion risk than clumping bamboo species in the continental United States. Journal for Nature Conservation, v.43, p.39-45, 2018. https://doi.org/10.1016/j.jnc.2018.02.012

LOU, Y.; SUN, H.; WANG, S.; XU, H.; LI, L.; \& ZHAO, H. ; Expression and functional analysis of two PsbS genes in bamboo (Phyllostachys edulis). Physiology Plantarum, 163, 459 - 471, 2018..https://doi.org/10.1111/ppl.12690

MUELLER-DOMBOIS, D.; ELLENBERG, H. Aims and methods of vegetation ecology. New York: John Wiley \& Sons; 1974, 547p.

R CORE TEAM. R: A language and environment for statistical computing. V. 4.0.4 (2021). R Foundation for Statistical Computing.

RONDON NETO, R.M.; KOZER, C.; ANDRADE, R. Do R. de; CECY, A.T.; HUMMES, P.A.; FRITZSONS, E.; CALDEIRA, M.V.W.; MACIEL, M.deN.M.; SOUZA, M.K.F. Caracterização florística e estrutural de um fragmento de floresta ombrófila mista, em Curitiba, PR - BR. Revista Floresta. Curitiba, v.32 n.1, p. 3-16, jan./jun. 2002.

SETTE JÚNIOR, C. R.; FONSECA LIMA, P. A.; MENDES LOPES, D. M.; GONDIM BARBOSA; CONEGLIAN, P, V,A,; ARAÚJO ALMEIDA, R. Characterization of biomass, charcoal and briquette of Phyllostachys aurea Carr. ex A. \& C. Rivière. Scientia Forestalis, Piracicaba, v. 45, n. 116, p. 619-628, dez/2017. DOI: dx.doi.org/10.18671/scifor.v45n116.03 
SPAKE, R.; SOGA, M.; CATFORD, J. A.; EIGENBROD, F. 2021. Applying the stressgradient hypothesis to curb the spread of invasive bamboo. Journal of Applied Ecology. 2021;00:1-11. DOI: 10.1111/1365-2664.13945

TIAN, X.K.; WANG, M.Y.; MENG, P.; ZHANG, J.S.; ZHOU, B.Z.; GE, X.G.; YU, F.H.; LI, M.H. Native Bamboo Invasions into Subtropical Forests Alter Microbial Communities in Litter and Soil. Forests, v.11, p.314, 2020; doi:10.3390/f11030314

WICKHAM, H. ggplot2: Elegant graphics for data analysis. Springer-Verlag.2016.

WANG, W.; SARDANS, J.; WANG, C.; ZENG, C.; TONG, C.; CHEN, G.; HUANG, J.; PAN, H.; PEGUERO, G.; VALLICROSA, $\mathrm{H}$. The response of stocks of $\mathrm{C}, \mathrm{N}$, and $\mathrm{P}$ to plant invasion in the coastal wetlands of China. Global Change Biology. 2018, 25, 733-743. DOI: $10.1111 /$ gcb.14491

XU, Q.F.; LIANG, C.F.; CHEN, J.H.; LI, Y.C.; QIN, H.; FUHRMANN, J. J. Rapid bamboo invasion (expansion) and its effects on biodiversity and soil processes. Global $\begin{array}{lllll}\text { Ecology and } & \text { Conservation } & 21 & \text { (2020) } & \end{array}$ https://doi.org/10.1016/j.gecco.2019.e00787

XU, Q.F.; JIANG, P.K.; WU, J.S. Bamboo invasion of native broadleaf forest modified soil microbial communities and diversity. Biological Invasions 17,433- 444 (2015). https://doi.org/10.1007/s10530-014-0741-y

ZHANG, H.; XUE, J. Spatial Pattern and Competitive Relationships of Moso Bamboo in a Native Subtropical Rainforest Community. Forests 2018, 9, 774; doi:10.3390/f9120774 